\title{
Stochastic Modelling of Solution Particle Movement: An Individual Case of Coupled Concentration Gradient Dependent and Independent Movements of Efavirenz
}

\author{
Tafireyi Nemaura ${ }^{1,2}$ \\ ${ }^{1}$ Department of Applied Mathematics, National University of Science and Technology, Bulawayo, Zimbabwe \\ ${ }^{2}$ Department of Clinical Pharmacology, University of Zimbabwe, Harare, Zimbabwe \\ Email: tnemaura@gmail.com, tafireyi.nemaura@nust.ac.zw
}

How to cite this paper: Nemaura, T. (2017) Stochastic Modelling of Solution Particle Movement: An Individual Case of Coupled Concentration Gradient Dependent and Independent Movements of Efavirenz. Journal of Applied Mathematics and Physics, 5, 1027-1034.

https://doi.org/10.4236/jamp.2017.55090

Received: March 30, 2017

Accepted: May 12, 2017

Published: May 16, 2017

Copyright $\odot 2017$ by author and Scientific Research Publishing Inc. This work is licensed under the Creative Commons Attribution International License (CC BY 4.0).

http://creativecommons.org/licenses/by/4.0/

\begin{abstract}
This work proposed a coupled model of diffusion. It adopted two forms of coupled movement, the interacting and non-interacting driven forms of movement of a solution particle of efavirenz concentration measured in blood plasma. Data from projected pharmacokinetics in a patient on efavirenz were used. A relationship between interacting and non-interacting diffusion was suggested through a stochastic differential equation. The solution particle with a small value of relative acceleration drift to its active neighbourhood was projected to have a corresponding high transport/interacting diffusion.
\end{abstract}

\section{Keywords}

Stochastic Differential Equation, Secondary Saturation Movement, Self Diffusion, Transport Diffusion

\section{Introduction}

The work attempted to combine two forms of diffusion starting with phenomenological approach motivated by Fick's laws and their mathematical solutions and the "random walk" of diffusing particles suggested by Robert Brown. This random walk of microscopic particles in suspension in a fluid has since been adopted as the "Brownian motion" in honour of Brown. The mathematical form of the Brownian motion was derived by Einstein [1]. The Fick's laws follow from Fourier's law of thermal conduction [1] [2] [3] [4].

The research encouraging modelling of random fluctuations found in PK/PD 
(Pharmacokinetic and Pharmacodynamic) relations has been suggested. It has been noted that there is an increasing need to extend the deterministic models which are currently favoured to models including a stochastic component in modelling pharmacological processes [5].

The transport diffusion is the concentration gradient dependent driven movement and describes the change in concentration of a solution particle. The transport diffusion can further be studied from Fick's laws by use of partial differential equations, however reseachers have used different variable spaces to study this flow [6] [7] [8]. Furthermore, in this work we tracked the transport diffusion by making use of the one to one function relationship between secondary saturation and concentration [9]. It is also noted that concentration is not a movement parameter as compared to secondary saturation. The self diffusion is a concentration gradient independent driven movement and describes the non-interacting movement of solution particle in the volume space. The observation of self diffusion component has been attributed to Brown [1].

The work has allowed for a proposition on the possibility to study the two movements in the $24 \mathrm{~h}$ dosing period that aid the process of diffusion by considering stochastic differential equations [5] [10]. It describes the movement of an interacting particle. Inferences were proposed on the possible relationship between the two movements (interacting and non-interacting). Numerical solutions on diffusional constituent movement characterisation were derived for a patient on efavirenz. This work presents a stochastic coupled diffusion model. This enables us to study the relationship between transport and self forms of diffusion. Stochastic coupled models have been applied on combined PK/PD processes [5]. In addition, in this case the coupling is in modelling the relationship of the two separate diffusional movements. A measure is developed to estimate the extent of gradient driven diffusion with the aid of a coupled stochastic differential equation. The Verhulst model is proposed to estimate the logistic relation obtained from modeling the self and transport forms of diffusion [11].

\section{Methods}

Simulated projected data on secondary saturation movement, time and concentration was taken from pharmacokinetic projections made on Patient $\mathrm{P}$ on 600 mg dose considered in Nemaura [9] [12]. Stochastic differential equations, Ordinary differential equations and linear and non-linear regression models are used to model diffusion in plasma. The following softwares, MATLAB and $\mathrm{R}$ were used for development of models. The solution of the Stochastic Differential Equations are approximated with the Euler-Maruyama scheme.

\subsection{Deterministic Models for Concentration and Gradient Driven Movements}

Initially there was modelling of the concentration profile of a solution particle of patient $\mathrm{P}$ in time. Letting $x(t)$ model concentration of solution particle at time $t$ and was given by, 


$$
\frac{\mathrm{d} x(t)}{\mathrm{d} t}=\theta(t),
$$

for some $\theta(t)$. The process $x(t)$ was sufficiently modelled by the following equation,

$$
x(t)=q t \mathrm{e}^{-r t} .
$$

We modelled the gradient driven movement $\left(x_{G}\right)$ by initially considering, the derived secondary saturation movement $(F(x, t))$, for some patient $\mathrm{P}$, in Nemaura (2014) from relations found which were informed from Nemaura (2015). The diffusant was the drug efavirenz in blood plasma. We gave the estimation of the secondary saturation movement in patient $\mathrm{P}$ with respect to time and concentration,

$$
F(x, t)= \begin{cases}\lambda_{1}\left(\mathrm{e}^{-\lambda_{2} t}-\mathrm{e}^{-\lambda_{3} t}\right), t \in[0,24]:=A, & \text { (i) } \\ \frac{u x}{v+x}, & x \in\left[0, c_{\max }\right]:=B .\end{cases}
$$

The following was noted,

$$
\frac{\mathrm{d} x_{G}}{\mathrm{~d} t}=\frac{\mathrm{d} x}{\mathrm{~d} F} \frac{\mathrm{d} F}{\mathrm{~d} t},
$$

where $x_{G}:=x(F(t))=x(t)$. Thus,

$$
\mathrm{d} x_{G}=\frac{\mathrm{d} x}{\mathrm{~d} F} \frac{\mathrm{d} F}{\mathrm{~d} t} \mathrm{~d} t=\frac{\mathrm{d} x}{\mathrm{~d} F} F^{\prime}(t) \mathrm{d} t,
$$

and,

$$
\frac{\mathrm{d} F}{\mathrm{~d} x}=1 / \frac{\mathrm{d} x}{\mathrm{~d} F}
$$

\subsection{Stochastic Model}

The secondary saturation movement followed a one to one relation with concentration and was thus used as compared to other forms of secondary movement that is convection and advection [9]. The process $y(t)\left(h^{-1}\right)$ modelled both the interaction and non-interacting diffusion movement of the solution particle in the volume space. The process $y(t)$ modelled these two diffusion forms of the solution particle and was thus given by,

$$
\mathrm{d} y(t)=\underbrace{\mathrm{d} x_{G}}_{M_{D}}+\underbrace{\mu(t, x(t)) \mathrm{d} t}_{M_{I}}+\overbrace{\sigma(t, x(t)) \mathrm{d} b(t)}^{E_{\epsilon}} y(0)=0,
$$

where the process $b(t)$ modelled the independent standard Brownian motion (Weiner process), $\mu(t, x(t))\left(h^{-2}\right)$ was the relative acceleration drift of a solution particle that allowed for the exchange of concentration material to its neighbourhood, $M_{D}$-auxilliary concentration gradient dependant driven movement, $M_{I}$-auxilliary concentration gradient independant driven movement and $E_{\epsilon}$-as the random term in $M_{I}$. Additionally,

$$
\mu(t, x(t)) \leq m,
$$

where at $\mu(t, x(t))=m, \rho(y(t), x(t))=0$ for $x(t)>0$. The parameter, $m$ is 
the least upper bound of drift for possible positive gradient driven process to occur. Thus the following:

$$
\begin{gathered}
\mathrm{d} y(t)=\frac{(v+x(t))^{2}}{u v} \mathrm{~d} F(t)+\mu(t, x(t)) \mathrm{d} t+\sigma(t, x(t)) \mathrm{d} b(t) \quad y(0)=0, \\
\mathrm{~d} y(t)=\left(\frac{(v+x(t))^{2}}{u v} F^{\prime}(t)+\mu(t, x(t))\right) \mathrm{d} t+\sigma(t, x(t)) \mathrm{d} b(t), \\
\mathrm{d} y(t)=\varphi(t, x(t)) \mathrm{d} t+\sigma(t, x(t)) \mathrm{d} b(t),
\end{gathered}
$$

where,

$$
\varphi(t, x(t))=\left(\frac{(v+x(t))^{2}}{u v} F^{\prime}(t)+\mu(t, x(t))\right) .
$$

Furthermore, for $\varphi(t, x)$ and $\sigma(t, x)$ and $\forall t \in A \subset \mathbb{R}_{+}, \quad x, z \in B \subset \mathbb{R}_{+}$ and some $C<\infty$ the following conditions are satistified,

$$
\begin{gathered}
|\varphi(t, x)-\varphi(t, z)| \leq C|x-z|,|\sigma(t, x)-\sigma(t, z)| \leq C|x-z|, \\
|\varphi(t, x)| \leq C(1+|x|),|\sigma(t, x)| \leq C(1+|x|) .
\end{gathered}
$$

Conditions 13 and 14 allowed for the existence and uniqueness of solutions for Equation (11) [10].

\section{Results}

\subsection{Modelling Concentration and the Concentration Gradient Driven Movement in the Absence of Self Diffusion}

We estimated the parameters in Equation (1) for the concentration-time curve (Table 1).

Furthermore, we estimated the parameter values for the derived secondary saturation $F(x, t)$ with respect to time and concentration in Patient $\mathrm{P}$ (Table 2 and Table 3).

Table 1. Parameter estimates in modelling deterministic solution particle concentration.

\begin{tabular}{ccccc}
\hline Parameters & Estimate & Std Error & $t$ value & $\operatorname{Pr}(>|t|)$ \\
\hline$q$ & 2.7996445 & 0.0280670 & 99.75 & $<2 \times 10^{-16}$ \\
$r$ & 0.1168398 & 0.0007906 & 147.79 & $<2 \times 10^{-16}$ \\
\hline
\end{tabular}

Table 2. Parameter estimates in modelling saturation movement rate with respect to $t$ (Model (3(i))).

\begin{tabular}{ccccc}
\hline Parameters & Estimate & Std Error & t value & $\operatorname{Pr}(>|t|)$ \\
\hline$\lambda_{1}$ & 0.623106 & 0.012214 & 51.02 & $<2 \times 10^{-16}$ \\
$\lambda_{2}$ & 0.022465 & 0.001203 & 18.68 & $5.35 \times 10^{-15}$ \\
$\lambda_{3}$ & 0.439258 & 0.017354 & 25.31 & $<2 \times 10^{-16}$ \\
\hline
\end{tabular}


Table 3. Parameter estimates in modelling saturation movement rate with respect to $x$ (Model (3(ii))).

\begin{tabular}{ccccc}
\hline Parameters & Estimate & Std Error & $t$ value & $\operatorname{Pr}(>|t|)$ \\
\hline$u$ & 0.801936 & 0.005934 & 135.1 & $<2 \times 10^{-16}$ \\
$V$ & 5.624198 & 0.126684 & 44.4 & $<2 \times 10^{-16}$ \\
\hline
\end{tabular}

There was consideration of how the concentration $x(t)$ and movement $y(t)\left(h^{-1}\right)$ processes informed potential relationships. The relationship of the concentration gradient driven movement and concentration rate of change was established by observing the relationship between equation 1 and 7 . The following was initially considered where $M_{I}=0$ and with $\eta(t, x(t))=0$ and $\sigma(t, x(t))=0$. There were two types of movement considered that were postulated to contribute to diffusion. The part $M_{D}$ modelled the auxilliary transport constituent of diffusion and $M_{I}$ measures the auxilliary self aspect of diffusion. A high correlation value was obtained of $\rho(x(t), y(t))=0.9667 \quad$ (estimated the extent of transport diffusion) in the $24 \mathrm{~h}$ dose interval, where $x(t), y(t)$ were solutions of Equations (1) and (7) (Figure 1).

Informed by results herewith there was an adoption that transport diffusion was driven by concentration. Thus concentration could be used as a substitute parameter for transport diffusion.

\subsection{Stochastic Model: Modelling the Solution Particle Movement with Variable Relative Acceleration (Drift)}

The following case was considered where $\mu(t, x(t)) \geq 0$ and $0 \leq \sigma(t, x(t)) \leq 1$. However, it was projected that at $\mu=3.2 h^{-2}=m$ the solution particle movement $(y(t))$ had zero correlation with the concentration process $x(t)$. In the interval where $\mu(t, x(t)) \geq 3.2 h^{-2}$, a solution particle movement was projected to have no transport diffusion. A solution particle with increasing relative acceleration drift to its neighbour would have less magnitude of extent with respect to transport diffusion. However, a solution particle with low relative drift to its neighbour had a high potential of transport diffusion. That is transport diffusion was more pronounced in neighbouring particles with relatively low acceleration drift. A relationship of the correlation between $x(t)$ and $y(t)$, and the drift in self diffusion was proposed (Figure 2).

An equation for the logistic decay relationship [11] was proposed,

$$
\rho(x(t), y(t))=\frac{1}{1+k \mathrm{e}^{l \mu(t, x(t))}}, \quad 0 \leq \mu(t, x(t)) \leq 3.2,
$$

where $k$ and $I$ (decay) are constants (Table 4). We obtained an analogous parameter to the Malthusian parameter and had a value of

$$
\operatorname{lm}=3.99269 \times 3.2=12.776608>1 .
$$

where $m$ generally defines the 'carrying capacity' however in this case it was the value of supremum of acceleration drift that enables transfer of concentration material [11]. 


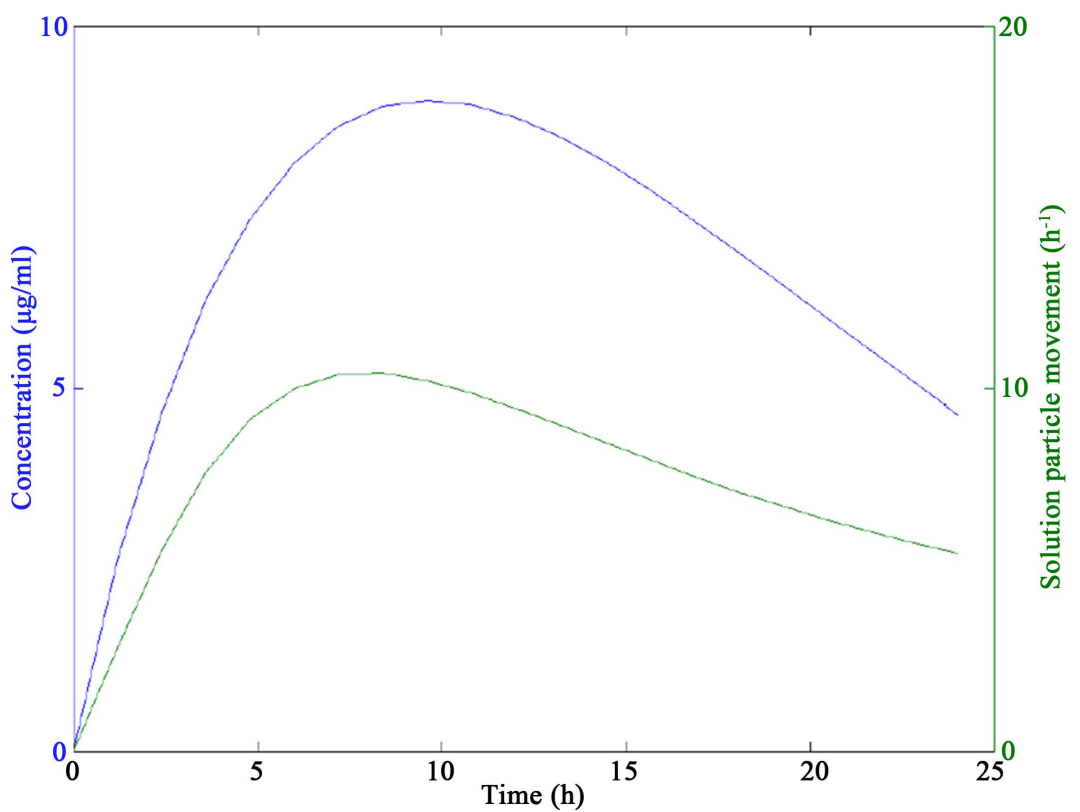

(a)

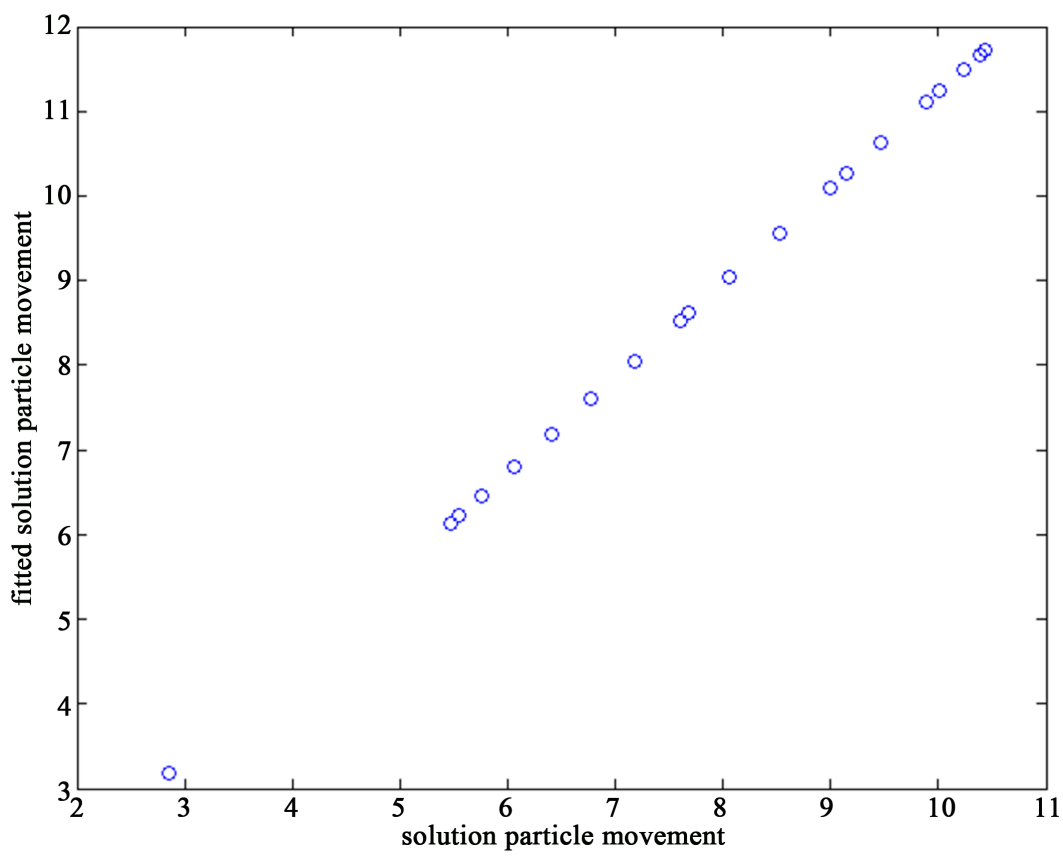

(b)

Figure 1. (a) Projected concentration and solution particle movement(transport diffusion with no self diffusion); (b) projected Estimated solution particle movement from the fitted equation $y(t)=1.1259 x(t)-0.0271$ and RMS value of 1.9554 .

Table 4. Parameter estimates in modelling solution particle concentration and solution particle movement correlation relationship in relation to relative acceleration drift.

\begin{tabular}{ccccc}
\hline Parameters & Estimate & Std Error & $\mathrm{t}$ value & $\operatorname{Pr}(>|t|)$ \\
\hline$l$ & 3.99269 & 0.25592 & 15.601 & $4.6 \times 10^{-14}$ \\
$k$ & 0.10301 & 0.01616 & 6.374 & $1.37 \times 10^{-6}$ \\
\hline
\end{tabular}




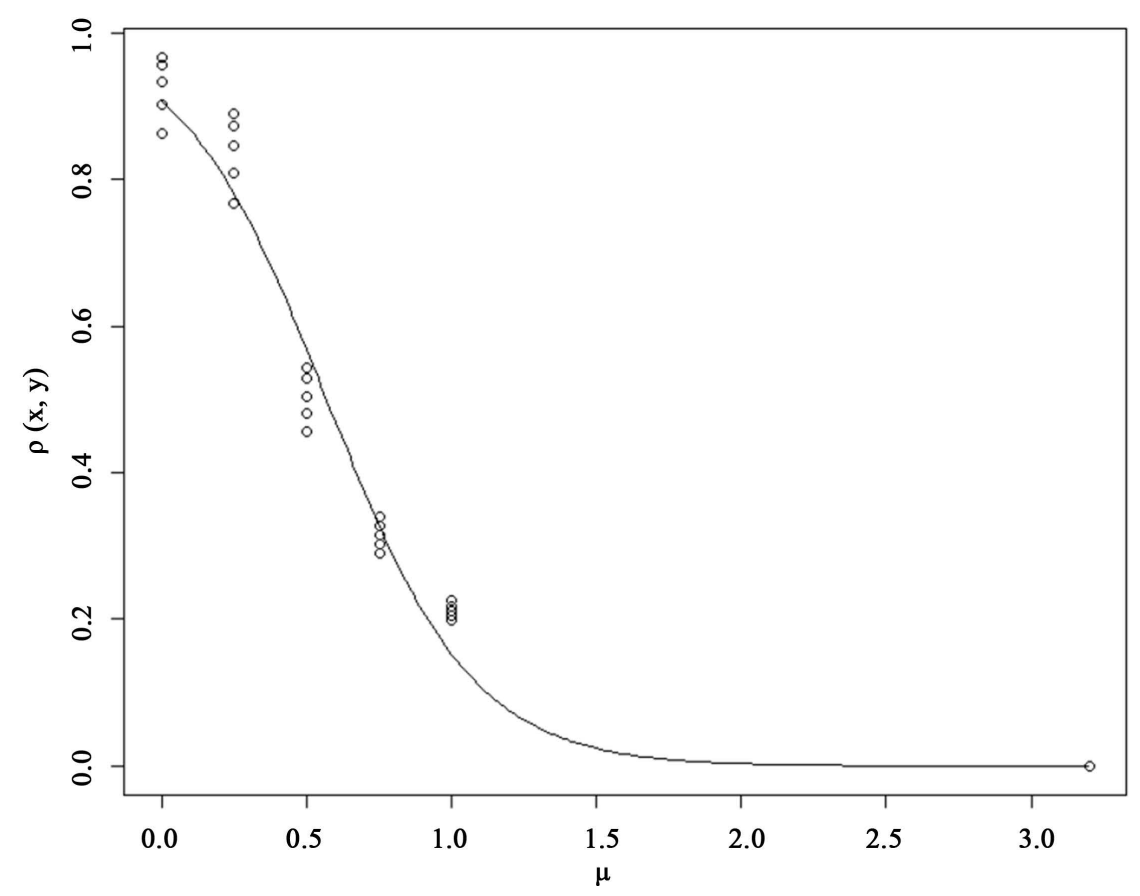

Figure 2. Projected solution particle concentration and solution particle movement correlation in relation to relative acceleration drift $\mu\left(h^{-2}\right)$ with $\sigma=0,0.25,0.5,0.75$ and 1 .

It is important to note that the relationship between concentration change and solution particle movement was inferred to be predominantly affected by relative acceleration drift.

\section{Conclusions}

Other researchers have been able to correlate self and transport forms of diffusion from experimental observations [8]. Transport diffusion has been shown to thrive in neighbouring solution particles that have zero relative drift. The transport diffusion in a neighbourhood of a solution particle was projected to be affected by drift. Considering an increased level of self diffusion of a solution particle relative to its active neighbourhood, showed that it will give out less in terms of concentration material to be exchanged through gradient. A logistic relation was projected between this correlation and drift. An elaborate study of the dynamics of the logistic decay curve proposed was done in Gonze, and considering the "Malthusian parameter value" resulted in an unstable steady state and unstable cycles for the system derived for Patient $\mathrm{P}$ since it had a value greater than 1 [11]. Relatively slow movement of neighbouring solution particles was projected to enable optimum drug transfer through transport diffusion in the plasma.

Throughout this work, there was an inherent assumption that the particles were already in orientation that enabled transport diffusion. This was because for transport diffusion to occur the particles should be in such an orientation which enables progression of that process [8]. The extent of the transport diffu- 
sion of a solution particle was projected to decrease with increasing relative drift to an active neighbourhood.

\section{Acknowledgements}

The author would like to thank the following; C. Nhachi, C. Masimirembwa, and G. Kadzirange, AIBST and The College of Health Sciences, University of Zimbabwe.

\section{References}

[1] Philibert, J. (2005) One and a Half Century of Diffusion: Fick, Einstein, before and Beyond. Diffusion Fundamentals, 2, 1-10.

[2] Fieldman, J. (2007) The Heat Equation (One Space Dimension). http://www.math.ubc.ca/ feldman/m267/heat1d.pdf

[3] Mehrer, H. and Stolwijk, N.A. (2009) Heroes and Highlights in the History of Diffusion. Diffusion Fundamentals, 11, 1-32.

[4] Narasimhan, T.N. (1999) Fourier's Heat Conduction Equation: History, Influence, and Connections. Reviews of Geophysics, 37, 151-172. https://doi.org/10.1029/1998RG900006

[5] Donnet, S. and Samson, A. (2013) A Review on Estimation of Stochastic Differential Equations for Pharmacokinetic/Pharmacodynamic Models. Advanced Drug Delivery Reviews, 65, 929-939.

[6] Nemaura, T. (2016) The Advection Diffusion-in-Secondary Saturation Movement Equation and Its Application to Concentration Gradient-Driven Saturation Kinetic Flow. Journal of Applied Mathematics and Physics, 4, 1998-2010. https://doi.org/10.4236/jamp.2016.411200

[7] Silvester, D.J., Griffiths, D.F. and Dold, J.W. (2015) Essential Partial Differential Equations: Analytical and Computational Aspects. Springer Undergraduate Mathematics Series, Springer Verlag, Heidelberg.

[8] Kärger, J. (2015) Transport Phenomena in Nanoporous Materials. ChemPhysChem, 16, 24-51. https://doi.org/10.1002/cphc.201402340

[9] Nemaura, T. (2015) Modeling Transportation of Efavirenz: Inference on Possibility of Mixed Modes of Transportation and Kinetic Solubility. Frontiers in Pharmacology, 6, 121. https://doi.org/10.3389/fphar.2015.00121

[10] Øksendal, B. (2003) Stochastic Differential Equations: An Introduction with Applications. 6th Edition, Springer, New York.

https://doi.org/10.1007/978-3-642-14394-6

[11] Gonze, D. (2015) The Logistic Equation. http://homepages.ulb.ac.be/dgonze/TEACHING/logistic.pdf

[12] Nemaura, T. (2014) Projections of Pharmacokinetic Parameter Estimates from Middose Plasma Concentrations in Individuals on Efavirenz; A Novel Approach. African Journal of Pharmacy and Pharmacology, 8, 929-952. 
Submit or recommend next manuscript to SCIRP and we will provide best service for you:

Accepting pre-submission inquiries through Email, Facebook, LinkedIn, Twitter, etc. A wide selection of journals (inclusive of 9 subjects, more than 200 journals)

Providing 24-hour high-quality service

User-friendly online submission system

Fair and swift peer-review system

Efficient typesetting and proofreading procedure

Display of the result of downloads and visits, as well as the number of cited articles Maximum dissemination of your research work

Submit your manuscript at: http://papersubmission.scirp.org/

Or contact jamp@scirp.org 